Siedlecki Zygmunt, Woźniak Jakub, Główczewska-Siedlecka Emilia, Śniegocki Maciej. Review of neurosurgical operative procedures in patients in the tenth decade of life. Journal of Education, Health and Sport. 2020;10(6):95-100. eISSN 2391-8306. DOI http://dx.doi.org/10.12775/JEHS.2020.10.06.010

https://apcz.umk.pl/czasopisma/index.php/JEHS/article/view/JEHS.2020.10.06.010

https://zenodo.org/record/3889926

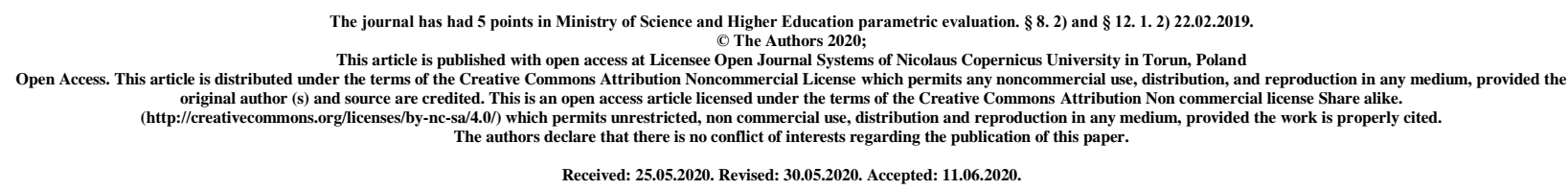

\title{
Review of neurosurgical operative procedures in patients in the tenth decade of life
}

\section{Zygmunt Siedlecki $^{1}$, Jakub Woźniak ${ }^{1}$, Emilia Glówczewska-Siedlecka ${ }^{2}$, Maciej Śniegocki ${ }^{1}$}

${ }^{1}$ Department of Neurosurgery, Neurotraumatology and Pediatric Neurosurgery, The Ludwik Rydygier Collegium Medicum in Bydgoszcz, The Nicolaus Copernicus University in Toruń

${ }^{2}$ Department of Geriatric, The Ludwik Rydygier Collegium Medicum in Bydgoszcz, The Nicolaus Copernicus University in Toruń

Running headline: neurosurgical procedures in elderly people

Corresponding address:

Dr. med. Zygmunt Siedlecki Department of Neurosurgery, Neurotraumatology and Pediatric Neurosurgery, the Ludwik Rydygier Collegium Medicum in Bydgoszcz, tel.: + 48606302680 e-mail: zs79@wp.pl 


\begin{abstract}
People over the age of 90 are hospitalized in neurosurgical departments, and the increase of life expectancy is conducive to such cases. Surgical treatment in older people is always at high risk, which may be due to the frailty syndrome that occurs. Qualification for surgical treatment of neurosurgical disorders in the elderly is not undertaken in the same way as in the younger population. This manuscript analyzes the patients in the 10th decade of life who were surgically treated at the neurosurgery clinic in 2019. It was shown that most of them were life threatening conditions. The most frequently operated disease was acute subdural hematoma, slightly less often chronic subdural hematoma and only a few cases of lumbar spine procedures. The analysis of patients treated surgically in comparison to patients not qualified for surgery because of other potentially neurosurgical diseases clearly indicates that surgical treatment of these patients is definitely rare.
\end{abstract}

Key words: neurosurgical procedures; elderly people

\title{
Introduction
}

Surgical treatment of elderly and advanced old age patients has an increased risk of severe perioperative complications [1,2]. It applies to every surgery of various anatomical regions in patients. Every surgical procedure, particular this in general anesthesia, induces important catabolic processes [2]. In the case of patients with burdens and comorbidities, surgery is at high risk of potentially fatal complications $[1,2]$. In the pathomechanism of aging, there is an increase in the activity of catabolic processes and their additional suspension by the operating procedures may prove fatal. In addition, functional reserves decrease in elderly diseases [3]. Such reserves decrese means that every stressor factor for example surgical procedure can lead to fatal and global decompensation of the whole organism [2,3]. Depletion of reserves is typical for frailty syndrome. It is believed that this syndrome occurs with increasing frequency with age. In the opinion of many authors, weakness syndrome occurs in $60 \%$ of patients after 80 years of age and as much as $80 \%$ of patients after 90 years of age [2]. This means that the vast majority of patients in the 10th decade of life meet the criteria of frailty syndrome. This syndrome was first described by Linda Fried, and its diagnostics is dealt with by geriatric departments [2,3]. Patients in the 10th decade of life who go to various surgical departments for various reasons: general surgery, orthopedics, neurosurgery and others should undergo careful analysis. When qualifying such patients for surgical or conservative treatment, the balance of potential benefits or complications arising from surgical treatment must be taken into account [4]. The specificity of patients in neurosurgical departments is that diseases and lesions requiring surgical treatment are located in important areas of the body - the central nervous system. In addition, surgical approach in neurosurgery is often causes traumatization of tissues - such as access to the spine. In addition, some spinal procedures require the use of artificial material implants [5]. Neurosurgical patients are often in severe clinical and functional condition due to brain or spinal cord disease. If advanced old age is added to this condition - any surgical intervention can be very aggravating and fatal to life. 


\section{Aim of the analysis}

The aim of this review was to assess patients in the 10th decade of life undergoing surgery, assess the indications for surgery, their condition, urgency of the procedure and the disease that had to be operated. The manuscript also evaluated the results of surgical treatment - functional status and mortality of patients. An assessment was also made of patients who at the same time were not qualified for surgery and were receiving conservative symptomatic treatment.

\section{Material and methods}

The study group were patients treated in Department of Neurosurgery, Neurotraumatology and Pediatric Neurosurgery of University Hospital No. 1, Collegium Medium in Bydgoszcz, Nicolaus Copernicus University in Torun in 2019, 8 patients over 90 years old were treated surgically, while 11 people in the 10th decade were treated conservatively. Conservatively treated patients were both hospitalized in the department and outpatient clinics. Both in the group of operated and conservatively treated patients, the vast majority were women. In the group of patients treated surgically there were 6 women aged 90-95 years $(\mathrm{Me}=92.83)$ and two men aged 90 and 92 years, while in the group of patients treated conservatively there were 10 women aged 90-97 years $(\mathrm{Me}=94.83)$ and one man aged 91 years. The gender distribution of the analyzed patients is presented in Table 1.

Table 1. Analyzed patients depending on gender

\begin{tabular}{|c|c|c|}
\hline & operative treatment & conservative treatment \\
\hline female & 6 & 10 \\
\hline male & 2 & 1 \\
\hline sum & 8 & 11 \\
\hline
\end{tabular}

An analysis of the underlying disease was carried out, which was the reason for admission to hospital and treatment of patients.

In the case of 8 patients treated surgically, medical diagnoses were:

- acute subdural hematoma (ASH) - 4 cases $(3 \mathrm{~F}, 1 \mathrm{M})$

- chronic subdural hematoma $(\mathrm{ChSH})$ - 3 cases $(2 \mathrm{~F}, 1 \mathrm{M})$

- degenerative stenosis of the lumbar spine - 1 case $(1 \mathrm{~F})$

In 11 patients treated conservatively the diseases were:

- degenerative spine disease with spinal stenosis - 4 cases $(4 \mathrm{~F})$

- vertebral compression fractures - 5 cases $(4 \mathrm{~F}, 1 \mathrm{M})$

- normotensive hydrocephalus - Hakim's syndrome - case (1F)

- subdural hygroma - 1 case $(1 \mathrm{~F})$

The patient was surgically treated in 7 cases and was admitted as a matter of urgency emergency service. In 4 patients treated for acute hematoma, they were qualified for immediate and life-saving surgery. In patients with chronic subdural hematoma, the patient's admission was also in emergency room, while the neurosurgical procedure did not take place immediately, but 
after prior preparation. In the case of one patient operated on due to osteoarthritis of the spine, the admission was planned and the patient was prepared for surgery by an anesthesiologist.

\section{Results}

Due to the small size of the study group, the results could not be statistically analyzed. In all 8 patients treated surgically, the most numerous group were patients operated on due to ASH. At admission, they were in a severe neurological condition, rated 3 - 8 in Glasgow Coma Scale (GCS) (for individual patients, respectively: 3, 5, 7, 8). Among these four patients operated on within 14 days of the postoperative period, 3 people died, while one patient whose condition was 8 GCS was discharged and transferred to a care and treatment facility in a functional state corresponding to 3 according to Glasow Outcome Scale (GOS). This patient was a significant success in the treatment process in our neurosurgical center. Of course, of all patients operated on for ASH, the survivor was in the best neurological condition.

Of the 3 patients operated on due to $\mathrm{ChSH}$, one patient died as a result of respiratory complications in the postoperative course, while two people were discharged home in a functional state comparable to that from before treatment in the hospital.

In total, of the 7 patients operated on due to intracranial hematomas ( $4 \mathrm{ASH}, 3 \mathrm{ChSH}), 4$ people died. Despite the lack of statistical attitudes, this means that the majority of patients in the 10th decade of life who were surgically treated for hematomas had an extremely poor prognosis.

One person treated surgically for lumbar stenosis with neurogenic claudication was qualified for surgery by the author of this work (ZS). The patient underwent anesthesiological assessment and then performed lumbar laminetomy without the use of any artificial implants. The patient had a $30 \%$ improvement in lower limb motility and objective radial symptoms. However subjectively, she complained of back pain and general pain, which made the clinical picture much less satisfactory.

Among the people conservatively treated for lumbar stenosis, the therapeutic effect was practically comparable when using the medication and rehabilitation. Among those treated conservatively for compression vertebral fractures, also due to dementia, no surgery was decided in these patients. However, in the author's opinion, a satisfactory level of improvement and patient satisfaction was achieved, using the right drugs and psychotherapy.

\section{Discussion}

Due to the fact that the society in Poland and the worldwide is aging and life expectancy is increasing, the issue of surgical treatment in old age or in severe old age is of interest to many authors and reports on this topic in references are available. Epstein N. E. et al. in 2011 issued a reference on spine surgery in geriatric patients [5]. In his work he noted that operating procedures in the spine of old people can be risky and can result in complications [5]. The frequency of these complications was calculated by Epstein et al. at 50-80\% [5]. In his manuscript, Epstein uses the term "unnecessary spine surgery" for situations where one neurosurgeon wants to operate a

geriatric patient's spine and another neurosurgeon does not want to. According to Epstein, such situations can result in poor postoperative outcome when the neurosurgeon decides to undergo 
surgical treatment [5]. Nagashima H. et al. In 2011 performed a retrospective study of 605 patients over 80 years of age who underwent surgery in 2004-2008 due to cervical stenosis with myelopathy [6]. On the basis of such a large study group, Nagashima estimated that the frequency of complications was not higher than in younger patients, and the search results were previously satisfactory. Nagashima estimated that after surgical treatment of geriatric patients it improves spinal content ranges by about $40 \%$ [6]. By Nagashima et al. surgical treatment of the spine in geriatric patients is optimistic [6], which is different from reports by Epstein et al. [6] and our data covered. It should be borne in mind, however, that in our neurosurgical department in Bydgoszcz in 2019 only one patient was operated on due to spinal stenosis, which does not allow to perform any conclusions. Lee D. H. et al. in 2010 published a manuscript on cardiac surgery in the elderly [7]. In this work, Lee improved that cardiac surgery has a significantly increased risk of complications and unfavorable prognosis [7]. Robinson T. N. et al. in his work had similar conclusions towards patients after colorectal surgery [8]. In his manuscript from 2011, he showed that colorectal surgery in geriatric patients is at increased risk compared to younger people [8].

\section{Conclusions}

Surgery in elderly patients, especially in severe old age is associated with an increased risk of complications and poor prognosis. Insofar as it is necessary in life-threatening situations, as in the case of intracranial hematomas, the decision about surgical treatment is obvious, although the postoperative course seems to be worse than in a younger person. However, if the surgery is planned - as in the case of spine surgery - the qualification for surgery must be undertaken by the neurosurgeon very carefully.

\section{Abbreviations}

- $\quad$ ASH - acute subdural hematoma

- $\quad \mathrm{ChSH}$ - chronic subdural hematoma

- GCS - Glasgow Coma Scale

- GOS - Glasow Outcome Scale

Declarations

- $\quad$ Ethics approval and consent to participate: Was not required because the work concerned only retrospective analysis of mecological documentation of patients without any participation or medical examination.

- $\quad$ Consent for publication: Written informed consent was not required.

- $\quad$ Availability of data and materials: All relevant data are within the paper.

- $\quad$ Competing Interests: The authors declare that they have no conflict of interest.

- $\quad$ Funding: The study was financed from own funds of the Neurosurgery of Collegium Medicum in Bydgoszcz. The authors received no specific founding for this work.

- Authors' contributions: 
Protocol/project development: ZS

Data collection and management: ZS, JW, E G-S

Data analysis: ZS, E G-S

Manuscript writing/editing: ZS

Verification of the manuscript in terms of substantive and formal: MS

All authors read and approved the final manuscript.

- Acknowledgements: the authors thank medical and nursing staff of neurosurgical department during the collection of medical data of patients being examined

\section{References}

1. Makary, M. A., Segev, D. L., Pronovost, P. J. et al. (2010). Frailty as a predictor of surgical outcomes in older patients. Journal of the American College of Surgeons, 210(6), 901-908.

2. Fried, L. P., Tangen, C. M., Walston, J. et al. (2001). Frailty in older adults: evidence for a phenotype. The Journals of Gerontology Series A: Biological Sciences and Medical Sciences, 56(3), M146-M157.

3. Kim, S., Brooks, A. K., \& Groban, L. (2015). Preoperative assessment of the older surgical patient: honing in on geriatric syndromes. Clinical interventions in Aging, 10, 13.

4. Robinson, T. N., Wu, D. S., Pointer, L., Dunn, C. L., Cleveland Jr, J. C., \& Moss, M. (2013). Simple frailty score predicts postoperative complications across surgical specialties. The American Journal of Surgery, 206(4), 544-550.

5. Epstein, N. E. (2011). Spine surgery in geriatric patients: Sometimes unnecessary, too much, or too little. Surgical neurology international, 2.

6. Nagashima, H., Dokai, T., Hashiguchi, H et al. (2011). Clinical features and surgical outcomes of cervical spondylotic myelopathy in patients aged 80 years or older: a multi-center retrospective study. European Spine Journal, 20(2), 240-246.

7. Lee, D. H., Buth, K. J., Martin, B. J., Yip, A. M., \& Hirsch, G. M. (2010). Frail patients are at increased risk for mortality and prolonged institutional care after cardiac surgery. Circulation, 121(8), 973.

8. Robinson, T. N., Wu, D. S., Stiegmann, G. V., \& Moss, M. (2011). Frailty predicts increased hospital and six-month healthcare cost following colorectal surgery in older adults. The American journal of surgery, 202(5), 511-514. 